\title{
EXTRAPOLATION OF AIRBORNE POLARIMETRIC AND INTERFEROMETRIC SAR DATA FOR VALIDATION OF BIO-GEO-RETRIEVAL ALGORITHMS FOR FUTURE SPACEBORNE SAR MISSIONS
}

\author{
Rolf Scheiber ${ }^{1}$, Seung-Kuk Lee ${ }^{1}$, Konstantinos P. Papathanassiou ${ }^{1}$, Nicolas Floury ${ }^{2}$ \\ ${ }^{1}$ German Aerospace Center (DLR), Microwave and Radar Institute, Germany \\ ${ }^{2}$ European Space Agency (ESA-ESTEC), Noordwijk-2200 AG, Netherlands
}

\begin{abstract}
This paper describes a methodology to extrapolate spaceborne quality SAR image products from long wavelength airborne polarimetric and interferometric SAR data. The methodology is applied to E-SAR data of DLR, partially acquired under ESA contract especially for the development and validation of bio/geo-retrieval algorithms in forested regions. For this purpose not only system (sensor) related parameters are altered, but also those relating to the propagation path (ionosphere) and to temporal decorrelation. Examples for future spaceborne products are presented and the potential of Pol-InSAR methods for the retrieval of forest heights from these data is discussed.
\end{abstract}

Index Terms - Delayed reflected signals, multi-path modeling, multi-path correction.

\section{INTRODUCTION}

Spaceborne SAR system concepts and mission design is often based on algorithms developed with and the experience gathered from airborne SAR data and associated dedicated campaigns. Airborne SAR systems have better performance parameters in terms of resolution, signal-tonoise ratio and ambiguity rejection than their future spaceborne counterparts as their design is not impacted by mass, power, and storage constraints. On the other hand, the scene coverage is limited due to the low flight altitude. Airborne SAR systems can be flexibly deployed to avoid huge temporal decorrelation as occurs in spaceborne mapping, where the interferometric revisit time is determined by the orbit choice, and ionospheric effects are not an issue. Due to these differences, extrapolation of data quality and algorithm performance form airborne to spaceborne scenarios can turn out to be a challenge.

In this paper we present a simulation approach which uses as input fully polarimetric airborne SAR data acquired in interferometric repeat-pass scenarios with very short temporal baselines, all acquisitions being performed within one hour. The data are deteriorated with respect to resolution, noise and ambiguity level to closely resemble the product specifications of future spaceborne SAR missions, like BIOMASS in P-band [1] or TerraSAR-L [2], Tandem-L [3] DESDinyl [4] in L-band. In addition controlled temporal decorrelation is imposed as well as Faraday rotation and ionospheric scintillations. The airborne data sets selected as input are representative for different forest scenarios (boreal, temperate, and tropical) and are used to validate different biomass estimation methods, which, using airborne data, have shown potential for a global above ground biomass estimation when applied to satellite SAR data, e.g. [5][6].

The paper is organized as follows. Section 2 presents in detail the airborne to spaceborne simulation strategy and section 3 shows comparative examples of the simulated data sets obtained from polarimetric data of DLR's airborne SAR sensor E-SAR. The performance of tree height retrieval based on Pol-InSAR methods is then exemplified in section 4. Section 5 concludes the paper.

\section{SIMULATION STRATEGY}

The individual simulation steps are shown in the block diagram of Fig.1. After controlled modification of the parameters determined by the SAR system design (resolution, noise and ambiguity level) other important characteristics of the data are modified in order to account for constraints imposed by the mission design, like the orbit height. In particular the impact of temporal decorrelation and ionospheric effects are simulated.

In the following the particularities for each simulation step are described. The simulation starts with the full resolution polarimetric scattering matrix data of an airborne acquisition. In case of interferometric slaves the data already co-registered to the master are used. The output of each simulation step serves as input to the next step. 


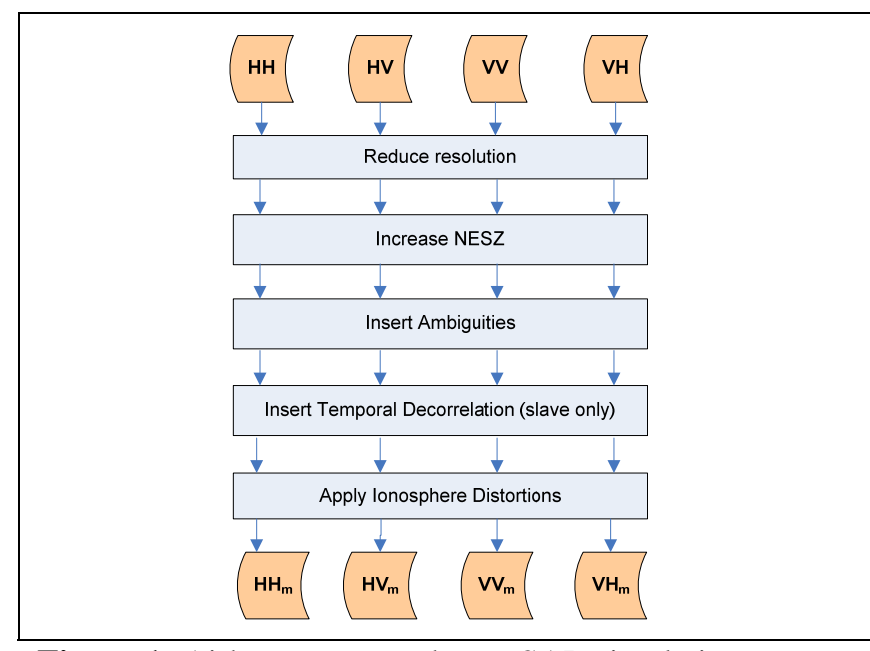

Figure 1: Airborne to spaceborne SAR simulation strategy.

Reducing the resolution: Airborne SAR data have improved resolution (1-2m slant-range resolution in the E-SAR case) compared to the specification of future spaceborne SAR products (slant-range resolutions of $25 \times 12.5 \mathrm{~m}$ in P-band and approx. $2 \times 7 \mathrm{~m}$ in L-band). Therefore the resolution is changed in a first step by reducing the range and azimuth signal bandwidth accordingly. At the same time, the spectral weighting is adjusted to ensure PSLR and ISLR of $25 \mathrm{~dB}$ and $10 \mathrm{~dB}$ respectively, according to specifications. Careful normalisation is required in order not to alter the backscattering coefficient $\sigma^{0}$ of the data.

Increasing the Noise-Equivalent Sigma-Zero (NESZ): The NESZ is determined by the radar equation. Due to the much higher observation range of orbital sensors compared to the airborne case this parameter is very critical for the sensor design and a considerable cost factor. As the NESZ is much higher in airborne data (in the order of $10 \mathrm{~dB}$ higher) it is sufficient to add random circular white Gaussian noise to the data according to the NESZ specification (for the examples of this paper a NESZ of $-28 \mathrm{~dB}$ is assumed). However, the noise spectrum must also be band-limited and weighted by the same function used for ensuring the resolution and the shape of the impulse response.

Inserting ambiguities: Much more than for the airborne case ambiguities are another critical design parameter in the space-borne context, especially with respect to the antenna size. The simulation strategy includes both azimuth and range ambiguities (20 $\mathrm{dB}$ signal to ambiguity ratio is assumed in both cases).

Azimuth ambiguities originate from the azimuth sidelobes of the antenna pattern and their position is determined by the PRF-time $t_{P R F}$ [7]:

$$
t_{P R F}=P R F \frac{\lambda r}{2 v^{2}},
$$

where $P R F$ is the pulse repetition frequency, $v$ is the effective velocity of the sensor, $\lambda$ is the wavelength and $r$ is the range. The relative azimuth and range positions are then computed as:

$$
\begin{gathered}
\Delta a z[m]= \pm t_{P R F} \cdot v, \\
\Delta r g[m]=\sqrt{r^{2}+v^{2} \cdot t_{P R F}^{2}}-r .
\end{gathered}
$$

The insertion of the front and aft azimuth ambiguities is performed as coherent addition of the attenuated and 2D shifted input data. In addition a small azimuth defocusing is also applied corresponding to the range displacement $\Delta \mathrm{rg}$. As scene extensions of airborne data are relatively small the computed shifts may be decreased by a factor of 10 to 100 .

Range ambiguities arise from distances considerable different than the imaged area and they are attenuated by the antenna elevation pattern. The range difference is determined by the PRF of the SAR instrument. Only the first ambiguity is assumed. In case of polarimetric SAR observations there is a toggling between the different transmit polarisations, which means that the ambiguities of one channel correspond to a different polarisation. Usually, cross-polarised channels are affected by co-polarised range ambiguity (and vice-versa). This property has been considered during simulations, and therefore the resulting amount of range ambiguities is much more severe in the HV and $\mathrm{VH}$ channels. Before coherently adding the range ambiguities, they were defocused, corresponding to the range difference, and attenuated by the nominal value.

Insertion of temporal decorrelation: In case of vegetation cover the coherence of interferometric pairs includes the so called volume decorrelation factor which is exploited by Pol-InSAR methods to derive vegetation height and extinction [6]. Unfortunately, orbital SAR sensors operate with repeat-cycles of 10 to 30 days, which make the data susceptible to temporal decorrelation. As volume and temporal decorrelation can hardly be separated it becomes important to analyze the impact of temporal decorrelation on the robustness of the inversion procedure.

A simple decorrelation model is assumed for controlled insertion of phase noise into interferometric slave images $s_{2}$ :

$$
S_{2, \text { temp_decorr }}(r, a)=\left|s_{2}(r, a)\right| \cdot\left[e^{j\left\langle s_{2}(r, a)\right\rangle}+N \cdot e^{j \theta_{\text {noise }}}\right]
$$

where the phase noise $\theta_{\text {noise }}$ is uniformly distributed in between $+/-\pi$, and $N$ is selected according to the desired decorrelation level. The relationship between the decorrelation factor $N$ and the temporal coherence contribution $\gamma_{\text {temp }}$ has been evaluated by simulations and is shown in Fig. 2. Although the adopted model in eq. 4 changes the magnitude of individual pixels, it does not change the mean magnitude and it does not insert additional noise, i.e. it does not change the NESZ. 


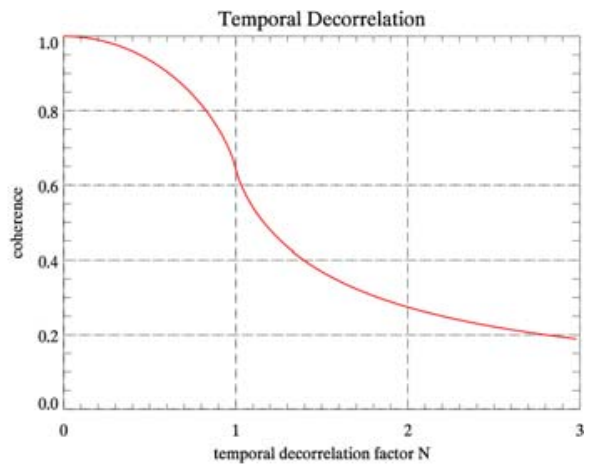

Figure 2: Mapping of coherence to decorrelation factor $N$.

Further, the inserted noise should not change the polarimetric signature and therefore $N$ and the $\theta_{\text {noise }}$ realization are assumed the same for all polarizations.

Applying ionospheric distortions: Ionospheric effects which impact orbital low frequency SAR data are Faraday rotation and the scintillation effect responsible for azimuth defocusing [8]. The ionospheric effects to alter the data have been obtained by numerical simulation using the parameterized WBMOD ionospheric model [9]. It uses a power law spectrum to generate a representation of the ionosphere for a certain geographic location, date, and time and compute the effective ionospheric phase screen. Different planetary indices and percentiles of occurrence are simulated. Fig. 3 shows such a phase screen (tangential to the local magnetic field). The screen has been rotated and projected to the slant-range plane of the SAR acquisition. Fig. 3 presents also the SAR acquisition geometry indicating the location of the ionospheric screen. The focused input SAR signal is first defocused in azimuth according to the range which corresponds to the ionospheric phase screen height (see Fig. 3). Then the phase screen is applied and the Faraday rotation is inserted.

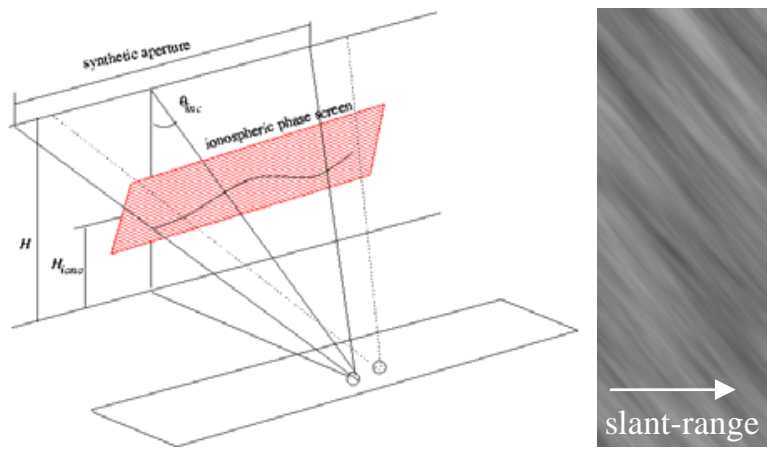

Figure 3: SAR acquisition geometry for insertion of ionospheric scintillations (left) and ionospheric phase screen transformed to the coordinates of the SAR acquisition (boreal location, $\mathrm{CkL}=90 \%, \mathrm{Kp}=1$, black to white $=2 \pi$ ).

Finally the data are refocused resulting in polarimetric data distorted by Faraday rotation and with defocusing corresponding to the scintillation strength.

\section{SIMULATION RESULTS}

The selected polarimetric and interferometric data sets for this study have been acquired by the E-SAR sensor in Land P-band during a campaign over tropical rain forest in Indonesia [6], and over boreal forest in Sweden.

An example of space-borne simulated interferometric L-band data with controlled insertion of temporal decorrelation is presented in Fig. 4 according to TerraSAR$\mathrm{L}$ specification. The data were acquired in 2004 during the INDREX-II campaign in Kalimantan, Indonesia. The area includes distorted (close to the river) and undistorted tropical rain forest. Another example is shown in Figure 5 for the P-band boreal case. Higher NESZ and ambiguity levels can be observed in the non-vegetated open areas.
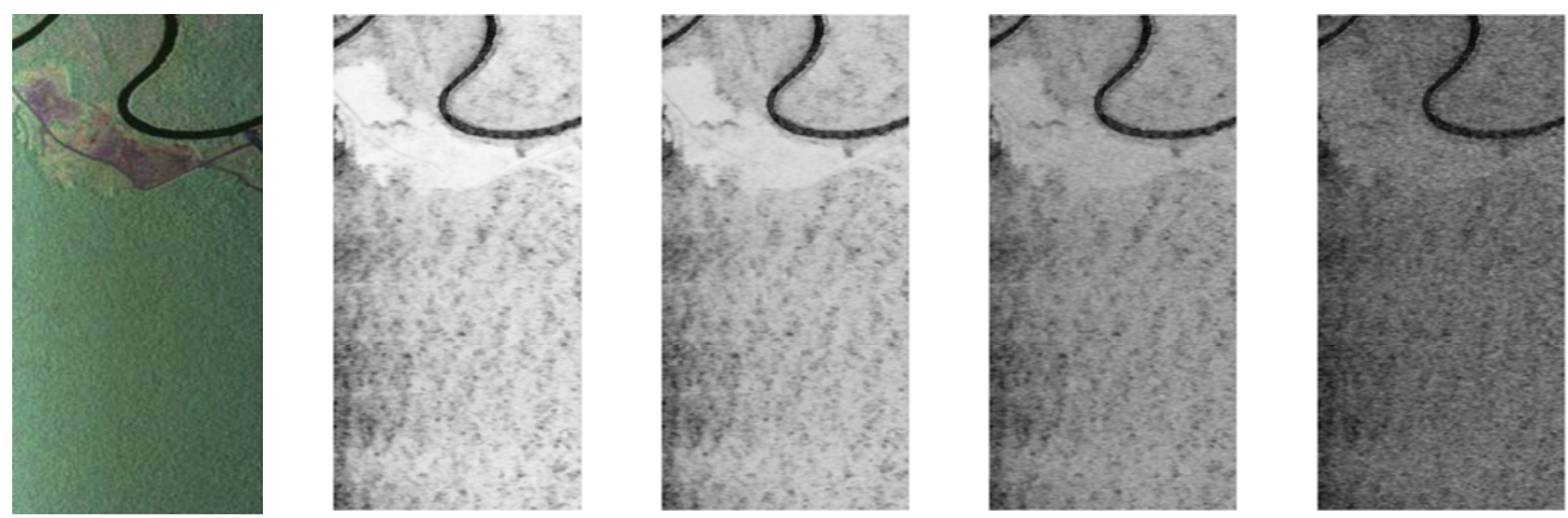

Figure 4: Controlled insertion of temporal decorrelation in L-band INDREX data: polarimetric color composite after spaceborne simulation, and four levels of coherence (unmodified original, and temporal coherence of 0.9, 0.7, and 0.5) 

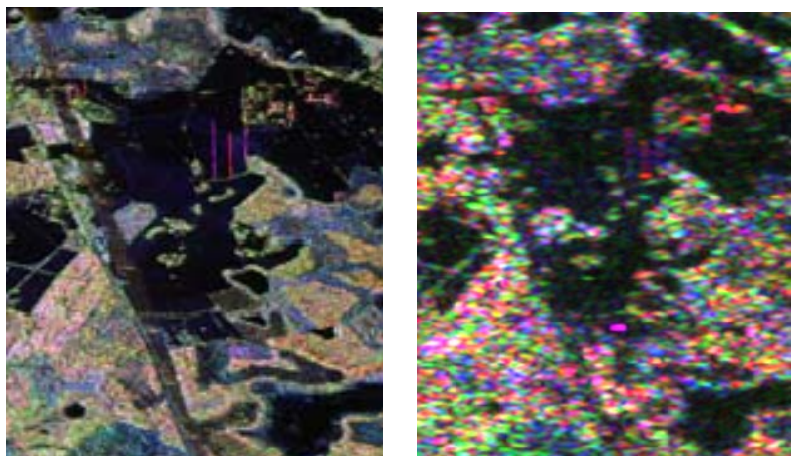

Figure 5: P-band polarimetric image (RGB=HH-HV-VV). Airborne input data (left) (ground resolution of $5 \mathrm{mx} 5 \mathrm{~m}$ ) and extrapolated spaceborne quality (right) (ground resolution of 50mx50m plus additional noise and ambiguities)

\section{COMPARATIVE FOREST HEIGHT INVERSION}

The forest height in the boreal test site (Remningstorp, Sweden) is estimated using data simulated according to the BIOMASS specification. Fig. 6 shows the comparison of Pol-InSAR inversion results between airborne data and the simulated data (incl. resolution, noise and ambiguities level). There is a tendency that the inverted forest height from simulation data is higher than from the airborne data result (see Fig 6, left). After normalizing by the total number of samples for a given airborne inverted height, we can see that low forests are more affected by constraints imposed by mission design than high forests (see Fig. 6, right). For the investigated L-band scenarios, and in the absence of temporal decorrelation, good correspondence in terms of forest height inversion has been achieved between airborne and simulated spaceborne scenario. A detailed discussion on the comparison approach is given in [10] including further results also for the equatorial case.

\section{CONCLUSIONS}

The extrapolated example data sets generated by the simulation strategy described in section 2 are helpful to demonstrate the performance of several bio/geo-retrieval algorithms. Critical mission parameters can be identified and optimized, leading to a cost-efficient mission design for future spacebrone SAR sensors. A first comparative analysis for Pol-InSAR P-band inversion has been presented and optimisation of parameters is in progress.

\section{ACKNOWLEDGEMENTS}

Special credits are given to S. Quegan and J.J. Green from University of Sheffield for providing the ionospheric phase screens used in this study. The work has been funded under ESA-ESTEC contract 21131/07/NL/LvH.
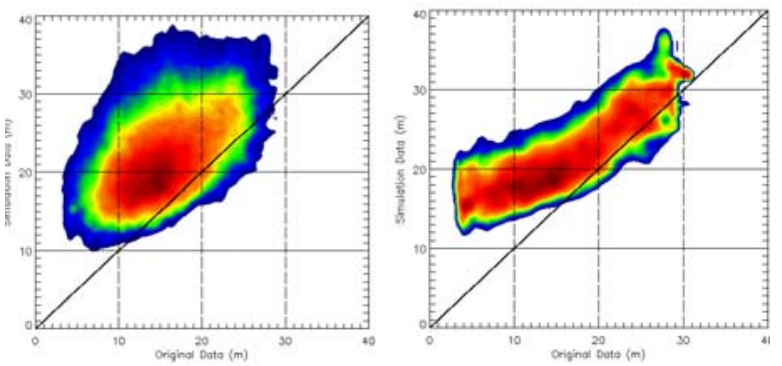

Figure 6: P-band Pol-InSAR inversion height comparison between airborne data (94 MHz bandwidth) and simulation data (6 $\mathrm{MHz}$ bandwidth plus additional noise and ambiguities). 2-dimensional histogram (left), 2-dimensional histogram normalized along column line (right).

\section{REFERENCES}

[1] ESA-ESTAC: "BIOMASS- report of assessment", http://www.esa.int/esaLP/LPfuturemis.html, 2008

[2] Torres, R. Lokas, S. Moller, H.L. Z Zink, M. Simpson, D.M.: "The TerraSAR-L mission and system"; Proc. IGARSS, Anchorage, 2004.

[3] Krieger G. et al: „The Tandem-L Mission Proposal: Monitoring Earth's Dynamics with High Resolution SAR Interferometry”, Proc. RadarCon, Pasadena, USA, May 2009.

[4] Freeman, A. et al, "Deformation, Ecosystem Structure, and Dynamics of Ice (DESDynI)," Proc. EUSAR 2008, Friedrichshafen, Germany, May 2008.

[5] Saatchi, S., Halligan, K., Despain, D., and Crabtree, R.L., "Estimation of forest fuel load from radar remote sensing", IEEE Transactions on Geoscience and Remote Sensing, 45, vol 6, 17261740, 2007.

[6] Hajnsek, I, Kugler, F. Lee, S.K. and Papathanssiou, K.P.: "Tropical-Forest-Parameter Estimation by Means of Pol-InSAR: The INDREX-II campaign”, IEEE Transactions on Geoscience and Remote Sensing, Vol. 47, No.2, pp.481-493, 2009

[7] I.G. Cumming and F.H. Wong, "Digital Processing of Synthetic Aperture Radar Data”, Artech House, ISBN: 1-58053058-3, Boston, 2005.

[8] Sh. Quegan, J.J. Green, K.P. Papathanassiou, R. Z. Schneider: "Quantifying and Correcting Ionospheric Effects on P-band SAR Images”, Proc. IGARSS, Boston, 2008.

[9] The WBMOD Ionospheric Scintillation Model: http://www.nwra-az.com/ionoscint/wbmod.html

[10] S.-K. Lee, F. Kugler, K. Papathanassiou, and I. Hajnsek, "Polarimetric SAR interferometry for forest application at P-band: potentials and challenges”, Proc. IGARSS 2009, Cape Town, South Africa, July 2009. 